\title{
Dynamic Analysis of a Tumor-Immune System under Allee Effect
}

\author{
Chunmei Zeng ${ }^{1,2}$ and Shaojuan Ma $\mathbb{D}^{1,3}$ \\ ${ }^{1}$ School of Mathematics and Information Science, North Minzu University, Yinchuan 750021, China \\ ${ }^{2}$ Yancheng Experimental Senior High School of ECNU, Yancheng 224002, China \\ ${ }^{3}$ The Key Laboratory of Intelligent Information and Big Data Processing of NingXia Province, North Minzu University, \\ Yinchuan 750021, China \\ Correspondence should be addressed to Shaojuan Ma; sjma@nmu.edu.cn
}

Received 9 June 2020; Accepted 18 August 2020; Published 2 September 2020

Academic Editor: Gisele Mophou

Copyright (C) 2020 Chunmei Zeng and Shaojuan Ma. This is an open access article distributed under the Creative Commons Attribution License, which permits unrestricted use, distribution, and reproduction in any medium, provided the original work is properly cited.

In this paper, we develop a definite tumor-immune model considering Allee effect. The deterministic model is studied qualitatively by mathematical analysis method, including the positivity, boundness, and local stability of the solution. In addition, we explore the effect of random factors on the transition of the tumor-immune system from a stable coexistence equilibrium point to a stable tumor-free equilibrium point. Based on the method of stochastic averaging, we obtain the expressions of the steady-state probability density and the mean first-passage time. And we find that the Allee effect has the greatest impact on the number of cells in the system when the Allee threshold value is within a certain range; the intensity of random factors could affect the likelihood of the system crossing from the coexistence equilibrium to the tumor-free equilibrium.

\section{Introduction}

With the continuous improvement of living standards, individuals pay more attention to their own health. As one of the most harmful diseases to human health and life, malignant tumor has aroused wide concern in the world. At the same time, it has also become a research hotspot in academia. Thompson [1] discussed strategies to target YAP/TAZ activity in cancer and suggested that YAP and TAZ are important drivers of solid tumor growth, metastasis, and resistance to therapy. Du et al. [2] represented a first-time report of NSCLC-intrinsic PD-1 expression and a potential mechanism by which PD-1 blockade may promote cancer growth. Studies on ascorbic acid, which chemosensitize colorectal cancer cells and synergistically inhibit tumor growth, were presented in [3]. Chaoul et al. [4] found that the lack of MHC class II molecules was beneficial to the infiltration of T-cells into tumors, thus enhancing the control of tumor growth. Villalobos et al. [5] demonstrated the anticancer effect of SOCS1 in prostate cancer and identified its potential inhibitory mechanism. Volz et al. [6] studied a new family of potent TLR9 agonists, EnanDIM, and it can inhibit tumor growth.
Although there are some achievements on finding factors which can effectively inhibit or control tumor growth. What we should not ignore is that the human body itself has a mechanism that can resist tumor cells-the immune system. It can not only protect the body from various internal and external factors against the normal immune system but also defend the body from tumor interference so that the normal operation of the body tissues. This function of the immune system has urged mathematicians and biologists exert to the research on its dynamic properties. Adriana et al. [7] discussed the regulation of angiogenesis by innate immune cells in the tumor microenvironment, specific features, and roles of major players. Key steps for visualizing the complex interaction between malignant tumors and the immune system were proposed and discussed in [8]. Jin et al. [9] summarized the current understanding of autophagy and its regulation in cancer. Medzhitov and Janeway [10] introduced the role of innate immune mechanism in the development of organisms and illustrated that it is the first line of defense of human immunity. The dynamics between tumor and immune cells in microenvironment can be referred to [11-14]. Khajanchi [15] 
described how tumor cells evolve and survive after encounter with the immune system. Terry et al. [16] presented new insights on the role of EMT in tumor-immune escape.

At present, the research on tumor-immune system has obtained above results, and Katrin et al. [17] showed that tumors have the Allee effect. According to the study of literature [18], the Allee effect is a correlation between any component of individual fitness and the number or density of the same individual. Since the Allee effect has been shown to change the optimal control decisions and control costs and estimate the potential risks in ecology, Katrin et al. [17] believed that it was also crucial for tumor growth control. However, so far, it has been neglected in tumor growth and persistence. Therefore, under the excitation of the above research, we attempt to add the Allee effect on the basis of Pillis [19] model, study its dynamic behavior, and explore the influence of Allee effect on tumor cells.

The remainder of the paper is organized as follows. In Section 2, we develop a definite tumor-immune model with the Allee effect and analyze the property of the deterministic model, including the positivity, boundness, and local stability. Section 3 is devoted to discuss the tumor-immune model with the Allee effect, which is disturbed by white noise. We carry out numerical simulations in Section 4. In Section 5, we take a discussion and draw a conclusion.

\section{The Deterministic Tumor-Immune Model with Allee Effect}

2.1. Establishment of Deterministic Model. In 2003, Pillis and Radunskaya [20, 21] proposed a mathematical model to describe the relationship between the tumor and immune system:

$$
\left\{\begin{array}{l}
\frac{\mathrm{d} E}{\mathrm{~d} t}=s+\frac{\rho E T}{\alpha+T}-\beta_{1} E T-d_{1} E \\
\frac{\mathrm{d} N}{\mathrm{~d} t}=r_{1} N\left(1-b_{1} N\right)-\beta_{2} N T \\
\frac{\mathrm{d} T}{\mathrm{~d} t}=r_{2} T\left(1-b_{2} T\right)-\beta_{3} E T-\beta_{4} N T
\end{array}\right.
$$

Yet, for the tumor itself, it has its own optimal density in the process of growth and reproduction, whether it is too dense or too thin will have an inhibitory effect on its growth.
Based on this, we study the relationship between tumor cells, normal cells, and immune cells under the background of the Allee effect. The mathematical model is as follows:

$$
\left\{\begin{array}{l}
\frac{\mathrm{d} E}{\mathrm{~d} t}=s+\frac{\rho E T}{\alpha+T}-\beta_{1} E T-d_{1} E \\
\frac{\mathrm{d} N}{\mathrm{~d} t}=r_{1} N\left(1-b_{1} N\right)-\beta_{2} N T \\
\frac{\mathrm{d} T}{\mathrm{~d} t}=r_{2} T\left(1-b_{2} T\right)(T-m)-\beta_{3} E T-\beta_{4} N T
\end{array}\right.
$$

where $E$ represents the number of immune cells, $T$ is the amount of tumor cells, $N$ can be thought of the quantity of normal cells, $s$ and $d_{1}$ represent the resource constant and mortality of effector immune cells, respectively, and $\mathrm{m}$ is Allee threshold and satisfies the condition of $m \cdot b_{2}<1$. Since the presence of tumor cells would activate effector immune cells, this term is expressed as $(\rho E T / \alpha+T)$ by Michaelis-Menten expression. Competition between effector immune cells and tumor cells would lead to the death of two types of cells, which are expressed as $-\beta_{1} E T$ and $-\beta_{3} E T$. The maximum growth rate and environmental carrying capacity of normal cells are represented by $r_{1}$ and $b_{1}^{-1}$, respectively. Similarly, the tumor cells are expressed by $r_{2}$ and $b_{2}^{-1}$. $\beta_{2}$ and $\beta_{4}$ stand for the cell death rate caused by the competition between normal cells and tumor cells, respectively.

In this study, we assume that the tumor is homogeneous. And according to the physical meaning of each variable, the initial conditions of model (2) are

$$
\begin{aligned}
& E(0)=E_{0}>0, \\
& N(0)=N_{0}>0, \\
& T(0)=T_{0}>0 .
\end{aligned}
$$

\subsection{Positiveness and Boundedness of Solutions}

Theorem 1. For any $t$ greater than 0 , the solution of system (2) is positive and bounded under the initial conditions (3), when $d_{1}>\rho$.

Proof. (1) Positiveness: integrating both sides of formula (2), we have the following relations:

$$
\left\{\begin{array}{l}
E(t)=E_{0} \exp \left[\int_{0}^{t} \frac{\rho T(\xi)}{\alpha+T(\xi)}-\beta_{1} T(\xi)-d_{1} d \xi\right]+s \int_{0}^{t} \exp \left[\int_{0}^{t} \frac{\rho T(\xi)}{\alpha+T(\xi)}-\beta_{1} T(\xi)-d_{1} d \xi\right] \mathrm{d} \theta, \\
N(t)=N_{0} \exp \left[\int_{0}^{t} r_{1}\left(1-b_{1} N(\xi)\right)-\beta_{2} T(\xi) d \xi\right], \\
T(t)=T_{0} \exp \left[\int_{0}^{t} r_{2}\left(1-b_{2} T(\xi)\right)(T(\xi)-m)-\beta_{3} E(\xi)-\beta_{4} N(\xi) d \xi\right] .
\end{array}\right.
$$


The initial condition is $E(0)>0, N(0)>0, T(0)>0$. When $t>0$, it can be obtained from the above equation:

$$
\begin{gathered}
E(t)>0, \\
N(t)>0, \\
T(t)>0 .
\end{gathered}
$$

(2) Boundedness: considering a function $V=E(t)+N(t)+T(t)$ when $d_{1}>\rho$, according to equation (2), we can obtain that

$$
\begin{aligned}
\dot{V}= & s+\frac{\rho E T}{\alpha+T}-\beta_{1} E T-d_{1} E+r_{1} N\left(1-b_{1} N\right)-\beta_{2} N T+r_{2} T\left(1-b_{2} T\right)(T-m)-\beta_{3} E T-\beta_{4} N T \leq s \\
& +\frac{r_{1}}{4 b_{1}}+R+\eta-\min \left\{\beta_{1}, \beta_{2}, \beta_{3}, \beta_{4}\right\} V .
\end{aligned}
$$

Then, the above equation can be written as follows:

$$
\frac{\mathrm{d} V}{\mathrm{~d} t}+\tau V \leq \varphi
$$

where $\tau=\min \left\{\beta_{1}, \beta_{2}, \beta_{3}, \beta_{4}\right\}$ and $\varphi=s+\left(r_{1} / 4 b_{1}\right)+$ $R+\eta$, where $R$ is the maximum value obtained by $r_{2} T\left(1-b_{2} T\right)(T-m)$ within the range of $T(t)>0$ and $\eta$ is a positive number selected which is greater than the difference between $E+N+T$ and $(E+N) T$. We then have

$0<V(E, N, T)<\frac{\varphi}{\tau}(1-\exp (-\tau t))+V\left(E_{0}, N_{0}, T_{0}\right) \exp (-\tau t)$.

When $t \longrightarrow \infty, 0<V<(\varphi / \tau)$. This shows that, under initial conditions (3), the solution of system (2) is bounded.
2.3. Classification of Equilibrium Points. According to equation (2), we get the following three kinds of equilibrium points:

(1) Tumor-free equilibrium point: $P_{0}=\left(\left(s / d_{1}\right),(1 /\right.$ $\left.\left.b_{1}\right), 0\right)$.

(2) Coexisting equilibrium point: $P_{1}=\left(\left(\left(s a+s T^{*}\right) /\right.\right.$ $\left.\left.\left(\left(\beta T^{*}+d_{1}\right)\left(a+T^{*}\right)-\rho T^{*}\right)\right),\left(r_{1}-\beta_{2} T^{*} / r_{1} b_{1}\right), T^{*}\right)$, where $T^{*}$ satisfies the equation

$$
f\left(T^{*}\right)=T^{4}+B T^{3}+C T^{2}+D T+E=0,
$$

and the coefficients of equation (9) are

$$
\begin{aligned}
& B=\alpha-m-\frac{1}{b_{2}}+\frac{d_{1}-\rho}{\beta_{1}}+\frac{\beta_{2} \beta_{4}}{r_{1} r_{2} b_{1} b_{2}}, \\
& C=-m \alpha+\frac{\alpha d_{1}+m \rho-m d_{1}}{\beta_{1}}+\frac{m-\alpha}{b_{2}}+\frac{\rho-r_{2} d_{1}}{r_{2} b_{2} \beta_{1}}+\frac{\beta_{1} \beta_{4}\left(r_{1}-\alpha \beta_{2}\right)+\beta_{2} \beta_{4}\left(\rho-d_{1}\right)}{r_{1} r_{2} b_{1} b_{2} \beta_{1}}, \\
& D=\frac{\alpha d_{1} r_{1} r_{2} m b_{1}+r_{1} b_{1} \beta_{3} s \alpha+r_{1} \alpha d_{1} \beta_{4}}{r_{1} r_{2} b_{1} b_{2} \beta_{1}} .
\end{aligned}
$$

(3) Abnormal equilibrium point: $p_{2}=\left(\left(s / d_{1}\right), 0,0\right)$,

$$
f\left(T^{*}\right)=A_{1} T^{4}+B_{1} T^{3}+C_{1} T^{2}+D_{1} T+E_{1}=0,
$$
$P_{3}=\left(\left(s \alpha+s T^{*} /\left({ }_{1} T^{*}+d_{1}\right)\left(\alpha+T^{*}\right)-\rho T^{*}\right), 0, T^{*}\right)$, where $T^{*}$ is determined by the equation

and the coefficients of above equation are

$$
\begin{aligned}
& A_{1}=r_{2} b_{2} \beta_{1}, \\
& B_{1}=r_{2} b_{2} \alpha \beta_{1}+r_{2} b_{2} d_{1}-\rho r_{2} b_{2}-\beta_{1} r_{2}-m r_{2} b_{2} \beta_{1}, \\
& C_{1}=r_{2} b_{2} \alpha d_{1}-r_{2} \alpha \beta_{1}-r_{2} d_{1}+r_{2} \rho-m r_{2} b_{2} d_{1}+m \rho r_{2} b_{2}+m \beta_{1} r_{2}-m r_{2} b_{2} \alpha \beta_{1}, \\
& D_{1}=m r_{2} \alpha \beta_{1}+m r_{2} d_{1}-m r_{2} \rho-\alpha r_{2} d_{1}-\alpha m d_{1} r_{2} b_{2}+\beta_{3} s, \\
& E_{1}=\alpha s \beta_{3}+\alpha m r_{2} d_{1} .
\end{aligned}
$$


2.4. Stability of Equilibrium Points. In this section, we analyze the local stability of the equilibrium points. The calculation shows that the number of normal cells at the abnormal equilibrium point is zero, which is not consistent with the practical significance of biology, so we only discuss the local asymptotic stability of tumor-free equilibrium point and coexistence equilibrium point.

The Jacobian matrix $J$ of system (2) at the equilibrium point is as follows:

$$
\left[\begin{array}{ccc}
\frac{\rho T}{\alpha+T}-\beta_{1} T-d_{1} & 0 & \frac{\alpha \rho E}{(\alpha+T)^{2}}-\beta_{1} E \\
0 & -2 r_{1} b_{1} N+r_{1}-\beta_{2} T & -\beta_{2} N \\
-\beta_{3} T & -\beta_{4} T & {\left[-3 r_{2} b_{2} T^{2}+\left(2 m b_{2} r_{2}+2 r_{2}\right) T-m r_{2}-\beta_{3} E-\beta_{4} N\right]}
\end{array}\right]
$$

2.4.1. Stability of Tumor-Free Equilibrium Point $P_{0}$. The eigenvalues of Jacobian matrix at $P_{0}$ are

$$
\begin{aligned}
& \lambda_{1}=-d_{1}<0, \\
& \lambda_{2}=-r_{1}<0, \\
& \lambda_{3}=-m r_{2}-\frac{s \beta_{3}}{d_{1}}-\frac{\beta_{4}}{b_{1}} .
\end{aligned}
$$

And by calculation, the equilibrium solution $P_{0}$ is locally asymptotically stable if and only if $-\left(s \beta_{3} / r_{2} d_{1}\right)-$ $\left(\beta_{4} / r_{2} b_{1}\right)<m<\left(1 / b_{2}\right)$, while $P_{0}$ is the saddle point with index two when $m<-\left(s \beta_{3} / r_{2} d_{1}\right)-\left(\beta_{4} / r_{2} b_{1}\right)$.

2.4.2. Stability of Coexistence Equilibrium Point $P_{1}$. When the equilibrium point is $P_{1}$, the characteristic equation of matrix (13) at this point is

$$
|J-\lambda E|=\lambda^{3}+\left(r_{1}-C_{2}-A_{2}+\beta_{4} N^{*}-\beta_{2} T^{*}\right) \lambda^{2}+D_{2} \lambda+E_{2}=0,
$$

where $T^{*}$ satisfies equation (9), and

$$
\begin{aligned}
& A_{2}=\frac{\rho T^{*}}{\alpha+T^{*}}-\beta_{1} T^{*}-d_{1}, \\
& B_{2}=\left[\frac{\alpha \rho}{\left(\alpha+T^{*}\right)^{2}}-\beta_{1}\right]\left[\frac{s \alpha+s T^{*}}{\left(\beta T^{*}+d_{1}\right)\left(a+T^{*}\right)-\rho T^{*}}\right], \\
& C_{2}=-3 r_{2} b_{2} T^{*}+\left(2 m r_{2} b_{2}+2 r_{2}\right) T^{*}-m r_{2}-\frac{s \beta_{3}\left(a+T^{*}\right)}{\left(\beta T^{*}+d_{1}\right)\left(a+T^{*}\right)-\rho T^{*}}, \\
& D_{2}=\left(A_{2}-r_{1}\right)\left(C_{2}-\beta_{4} N^{*}\right)-r_{1} A_{2}+\left(A_{2} \beta_{2}+B_{2} \beta_{3}+C_{2} \beta_{2}\right) T^{*}-2 \beta_{2} \beta_{4} N^{*} T^{*}, \\
& E_{2}=r_{1} A_{2} C_{2}-B_{2} \beta_{2} \beta_{3} T^{*}-A_{2} C_{2} \beta_{2} T^{*}-A_{2} \beta_{4} r_{1} N^{*}+B_{2} r_{1} \beta_{3} T^{*}+2 A_{2} \beta_{2} \beta_{4} N^{*} T^{*}, \\
& N^{*}=\frac{r_{1}-\beta_{2} T^{*}}{r_{1} b_{1}} .
\end{aligned}
$$

According to Routh-Hurwitz criterion, we know that

$$
\begin{aligned}
& a_{0}=1 \\
& a_{1}=r_{1}+m r_{2}+d_{1}+(3-2 m) r_{2} b_{2}+\left(\beta_{1}-2 r_{2}-\frac{\rho}{\alpha+T^{*}}\right) T^{*}+\frac{r_{1}-\beta_{2} T^{*}}{r_{1} b_{1}} \beta_{4}+\left[\beta_{3}-\frac{\alpha \rho T^{*}}{\left(\alpha+T^{*}\right)^{2}}+\beta_{1} T^{*}\right]\left[\frac{s \alpha+s T^{*}}{\left(\beta T^{*}+d_{1}\right)\left(a+T^{*}\right)-\rho T^{*}}\right] \\
& a_{2}=\left(A_{2}-r_{1}\right)\left(C_{2}-\frac{r_{1}-\beta_{2} T^{*}}{r_{1} b_{1}} \beta_{4}\right)-r_{1} A_{2}+\left(A_{2} \beta_{2}+\beta_{3} B_{2}+C_{2} \beta_{2}\right) T^{*}-2 \beta_{2} \beta_{4} T^{*} \frac{r_{1}-\beta_{2} T^{*}}{r_{1} b_{1}} \\
& a_{3}=\left(r_{1}-\beta_{2} T^{*}\right) A_{2} C_{2}+\left(r_{1}-\beta_{2}\right) B_{2} \beta_{3} T^{*}+\left(2 \beta_{2} T^{*}-r_{1}\right) \frac{r_{1}-\beta_{2} T^{*}}{r_{1} b_{1}} A_{2} \beta_{4} .
\end{aligned}
$$


The determinant of Hurwitz can be calculated according to the above expressions:

$$
\begin{aligned}
\Delta_{1} & =a_{1}, \\
\Delta_{2} & =\left|\begin{array}{ll}
a_{1} & a_{0} \\
a_{3} & a_{2}
\end{array}\right|, \\
\Delta_{3} & =\left|\begin{array}{lll}
a_{1} & a_{1} & 0 \\
a_{3} & a_{2} & a_{1} \\
0 & 0 & a_{3}
\end{array}\right| .
\end{aligned}
$$

Thus, when $\Delta_{1}>0, \Delta_{2}>0$, and $\Delta_{3}>0$ are satisfied at the same time, coexistence balance $P_{1}$ is locally asymptotically stable.

By equation (9), we know that coexistence equilibrium expression $T^{*}$ is the root of quartic equation, namely, there are at most four coexistence equilibrium points. Considering the meaningful number of equilibrium and its stability is related to parameter selection, and in order to make the results more intuitive, we carry out numerical simulation according to effective range of variables. As a result, the number and stability of the coexistence equilibrium point are shown in Figure 1. In this numerical simulation, the horizontal axis is $\rho$ value from 0 to 2 , while the vertical axis is $m$ value from -2 to 1 .

In order to reveal the role of the Allee effect in the system, we only change the value of $m$. By referring to $[20,21]$, we make $\rho=0.01$; then, the time history diagrams of three cells in different $m$ are as follows.

Observing Figure 2, we know when $m \in(-\infty,-1.2)$ $\cup(-0.5,1)$, the number of the three types of cells do not change significantly. When $m$ belongs to $(-1.2,-0.5)$, the cell change trend is the most significant. This indicates that when the Allee threshold of tumor cells is within the range of -1.2 to -0.5 , the Allee effect has the greatest influence on the number of cells in the system, and this also proves that the Allee effect has an impact on the tumor-immune system.

\section{Stochastic Tumor-Immune Model with Allee Effect}

3.1. Establishment of Stochastic Model. Considering that the growth of tumor cells can be affected by various uncertainties, such as vibration and temperature, and these can be simulated with noise, therefore, based on the deterministic model (2), we propose the following stochastic model:

$$
\left\{\begin{array}{l}
\dot{E}_{1}=s+\frac{\rho E_{1} T_{1}}{\alpha+T_{1}}-\beta_{1} E_{1} T_{1}-d_{1} E, \\
\dot{N}_{1}=r_{1} N_{1}\left(1-b_{1} N_{1}\right)-\beta_{2} N_{1} T_{1}+N_{1} W_{1}(t), \\
\dot{T}_{1}=r_{2} T_{1}\left(1-b_{2} T_{1}\right)\left(T_{1}-m\right)-\beta_{3} E_{1} T_{1}+\beta_{4} N_{1} T_{1} \\
+T_{1} W_{2}(t) .
\end{array}\right.
$$

Among them, $E_{1}(t), N_{1}(t)$, and $T_{1}(t)$ are three random processes, which represent the number of immune cells, normal cells, and tumor cells, respectively. $W_{i}(t)$ is the independent Gaussian white noise with zero mean intensity $D_{i}(i=1,2)$, which acts on the natural growth rate of normal cells $\rho$ and the nature growth rate of tumor $r_{2}$, and we assume that both damping and excitation are small. The specific meanings of other parameters are the same as those in model (2).

3.2. The Stochastic Averaging Method. In order to facilitate the application of the stochastic average method, we first simplified model (19), setting

$$
\begin{aligned}
f & =-r_{2} m, g=r_{1} b, G_{3}=-\gamma_{1} b_{1} N_{1}, \\
G_{1}\left(E_{1}, N_{1}, T_{1}\right) & =\frac{s}{E_{1}}+\frac{\rho T_{1}}{a+T_{1}}-\beta_{1} T_{1}, \\
G_{2} & =r T\left(1-b_{2} T_{1}+b_{2} m\right)-\beta E .
\end{aligned}
$$

Then, model (19) can be written as follows:

$$
\left\{\begin{array}{l}
\dot{E}_{1}=E_{1}\left[-d_{1}+G_{1}\left(E_{1}, N_{1}, T_{1}\right)\right], \\
\dot{N}_{1}=N_{1}\left[r_{1}-\beta_{2} T_{1}+G_{3}\left(E_{1}, N_{1}, T_{1}\right)\right]+N_{1} W_{1}(t), \\
\dot{T}_{1}=T_{1}\left[-\beta_{4} N_{1}+f+G_{2}\left(E_{1}, N_{1}, T_{1}\right)\right]+T_{1} W_{2}(t) .
\end{array}\right.
$$

The above equation (21) is converted into the following It $\hat{o}$ stochastic differential equation by Itô formula:

$$
\left\{\begin{array}{l}
\mathrm{d} E_{1}=E_{1}\left[-d_{1}+G_{1}\left(E_{1}, N_{1}, T_{1}\right)\right] \mathrm{d} t, \\
\mathrm{~d} N_{1}=N_{1}\left[r_{1}-\beta_{2} T_{1}+G_{3}\left(E_{1}, N_{1}, T_{1}\right)+\frac{1}{2} D_{1}\right] \mathrm{d} t \\
\quad+\sqrt{D_{1}} N_{1} \mathrm{~d} B_{1}(t), \\
\mathrm{d} T_{1}=T_{1}\left[f-\beta_{4} N_{1}+G_{2}\left(E_{1}, N_{1}, T_{1}\right)+\frac{1}{2} D_{2}\right] \mathrm{d} t \\
\quad+\sqrt{D_{2}} T_{1} \mathrm{~d} B_{2}(t),
\end{array}\right.
$$

where $B_{1}(t)$ and $B_{2}(t)$ are independent Wiener process and $(1 / 2) D_{1} E_{1} \mathrm{~d} t$ and $(1 / 2) D_{2} T_{1} \mathrm{~d} t$ are the Wong-Zakai amendments. Defining a random process,

$$
R\left(N_{1}, T_{1}\right)=\beta_{2} T_{1}-\beta_{4} N_{1}+f-r_{1}+f \ln \frac{\beta_{4} N_{1}}{f}-r_{1} \ln \frac{\beta_{2} T_{1}}{r_{1}} .
$$

Among them, $R\left(\left(f / \beta_{4}\right),\left(r_{1} / \beta_{2}\right)\right)=0 . \beta_{2}, \beta_{4}$, and $r_{1}$ are positive, $m<0$. Then, the Itô stochastic differential equation of random process $R(t)$ is 

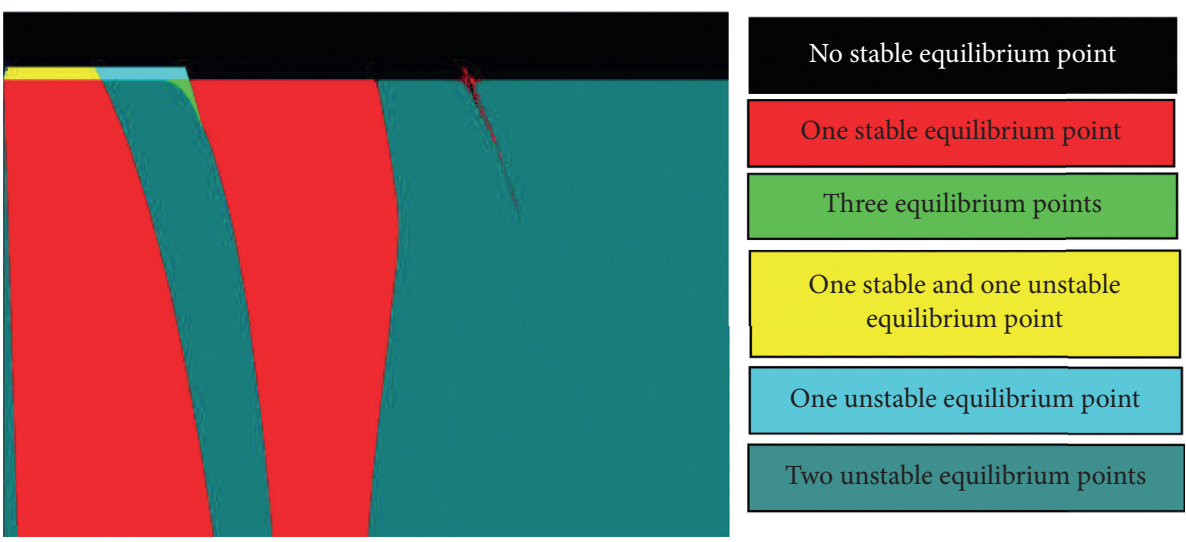

FIgURE 1: The number of coexistence equilibrium points and their stability.

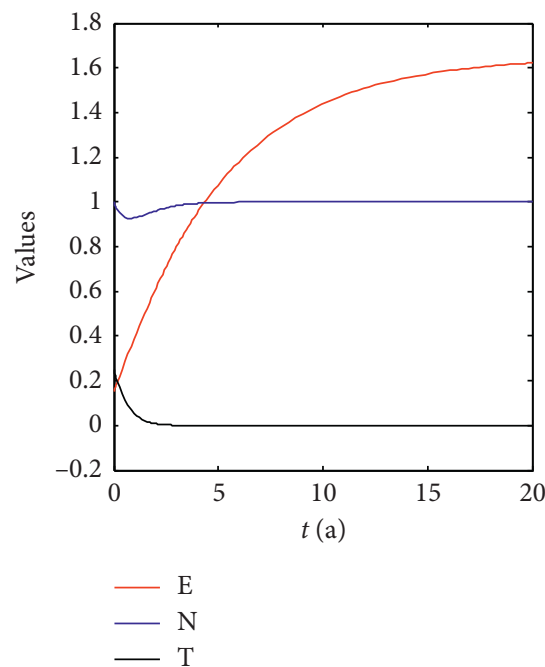

(a)

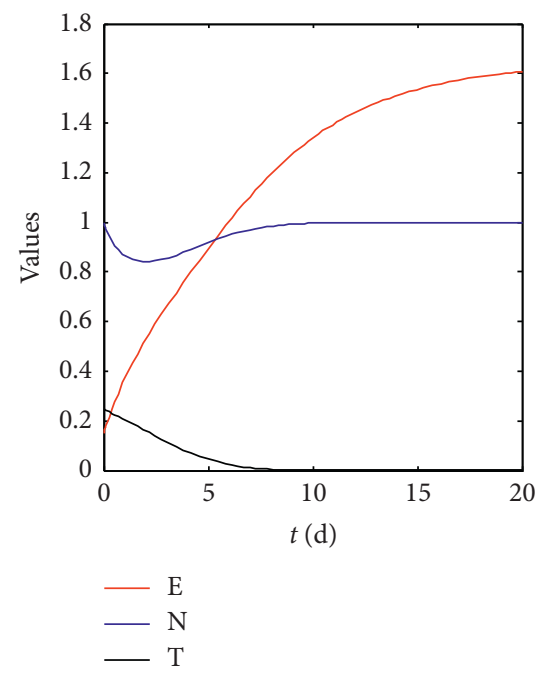

(d)

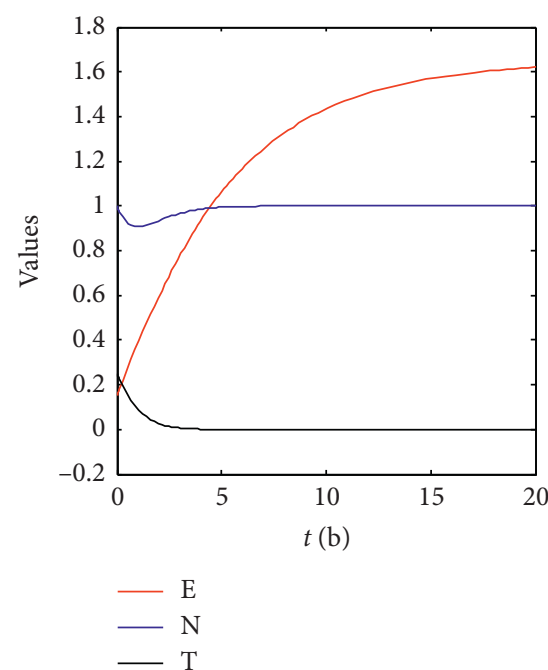

(b)

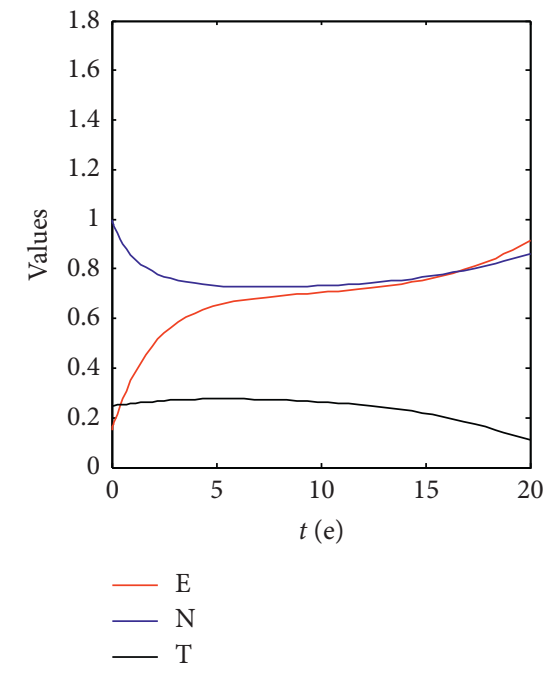

(e)

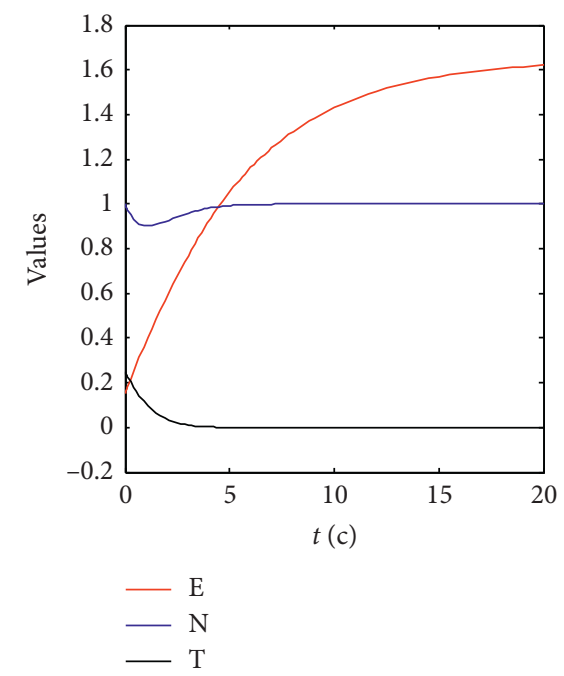

(c)

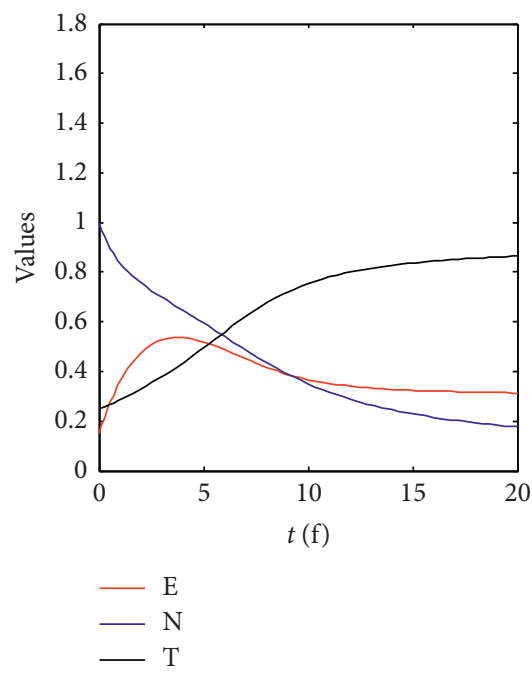

(f)

Figure 2: Continued. 


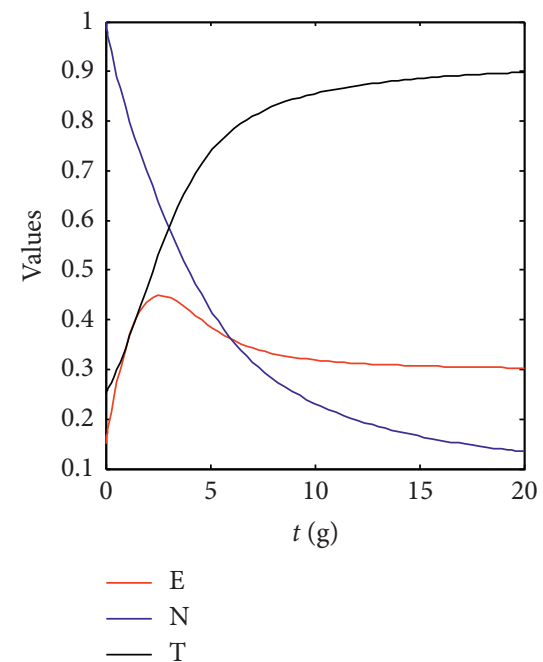

(g)

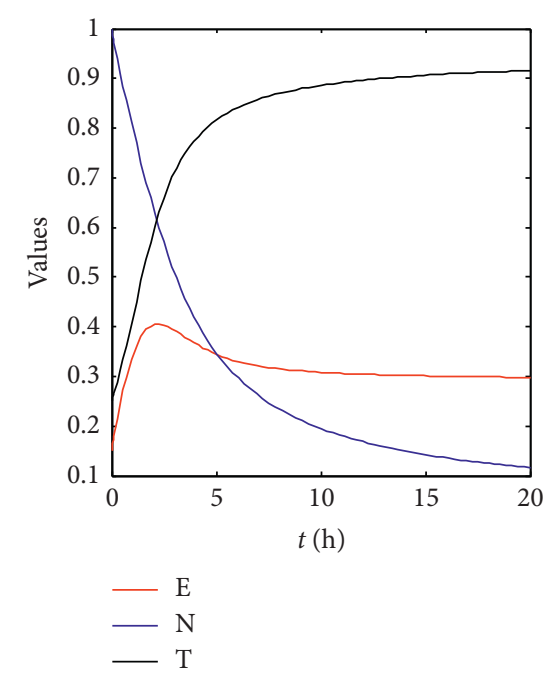

(h)
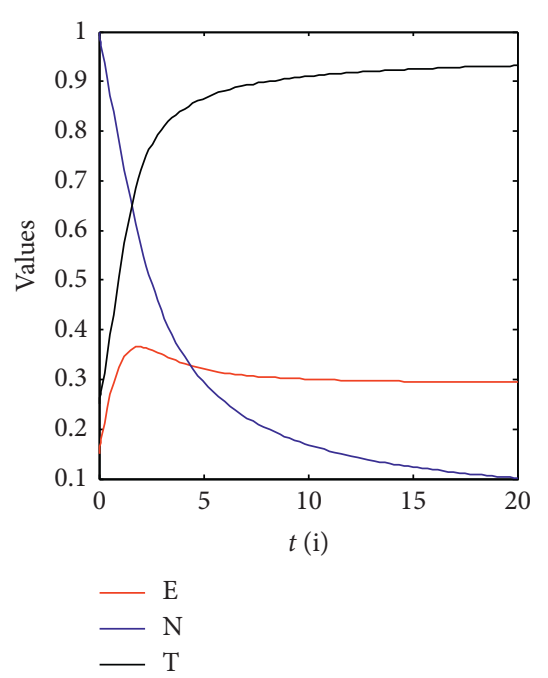

(i)

Figure 2: Time history diagram of different $m(\rho=0.01$ ). (a) $m=0.5$. (b) $m=0.1$. (c) $m=0.0$. (d) $m=-0.5$. (e) $m=-0.7$. (f) $m=-0.8$. (g) $m=-1.0$. (h) $m=-1.2$. (i) $m=-1.5$.

$$
\mathrm{d} R=\left[\left(f-\beta_{4} N_{1}\right) G_{3}+\left(\beta_{2} T_{1}-r_{1}\right) G_{2}-\frac{1}{2} \beta_{4} N_{1} D_{1}+\frac{1}{2} \beta_{2} T_{1} D_{2}\right] \mathrm{d} t+\sqrt{D_{1}}\left(f-\beta_{4} N_{1}\right) \mathrm{d} B_{1}+\sqrt{D_{2}}\left(\beta_{2} T_{1}-\gamma_{1}\right) \mathrm{d} B_{2} .
$$

$$
\mathrm{d} R=m(R) \mathrm{d} t+\sigma(R) \mathrm{d} B(t)
$$

Using the random average principle of StratorovichKhasminskii, the average Itô stochastic differential equation of $R(t)$ is

Whence,

$$
\begin{aligned}
& m(R)=\left\langle\left(f-\beta_{4} N_{1}\right) G_{3}+\left(\beta_{2} T_{1}-r_{1}\right) G_{2}\right\rangle_{t}+\frac{1}{2} \beta_{2} D_{2}\left\langle T_{1}\right\rangle_{t}-\frac{1}{2} \beta_{4} D_{1}\left\langle N_{1}\right\rangle_{t}, \\
& \sigma^{2}(R)=D_{1}\left\langle\left(f-\beta_{4} N_{1}\right)^{2}\right\rangle_{t}+D_{2}\left\langle\left(\beta_{2} T_{1}-r_{1}\right)^{2}\right\rangle_{t},
\end{aligned}
$$

and according to the formula,

$$
\langle\cdot\rangle_{t}=\frac{1}{T(R)} \oint[\cdot] \mathrm{d} t .
$$

We have the following expressions:

$$
\begin{aligned}
\left\langle N_{1}\right\rangle_{t} & =\frac{f}{\beta_{4}}, \\
\left\langle T_{1}\right\rangle_{t} & =\frac{r_{1}}{\beta_{2}}, \\
r_{1}\left\langle\left(f-\beta_{4} N_{1}\right)^{2}\right\rangle_{t} & =f\left\langle\left(r_{1}-\beta_{2} T_{1}\right)^{2}\right\rangle_{t}=\frac{g(R)}{T(R)},
\end{aligned}
$$

where

$$
g(R)=r_{1} \oint \frac{f-\beta_{4} N_{1}}{T_{1}} \mathrm{~d} T_{1}
$$

Then, with the help of equations (26)-(30), the drift coefficient and diffusion coefficient can be simplified as follows:

$$
\begin{aligned}
m(R) & =\frac{1}{2} r_{1} D_{2}-\frac{1}{2} f D_{1}-b_{1} \frac{g(R)}{T(R)}, \\
\sigma^{2}(R) & =\frac{f D_{1}-r_{1} D_{2}}{r_{1} f} \frac{g(R)}{T(R)} .
\end{aligned}
$$

3.2.1. Stationary Probability Density. Base on equation (23), we obtain that

$$
\begin{aligned}
& \ln \frac{\beta_{4} N_{1}}{f} \approx 1+\frac{\beta_{4} N_{1}-f}{f}-\frac{1}{2}\left(\frac{\beta_{4} N_{1}-f}{f}\right)^{2}, \\
& \ln \frac{\beta_{4} N_{1}}{r_{1}} \approx 1+\frac{\beta_{2} T_{1}-r_{1}}{r_{1}}-\frac{1}{2}\left(\frac{\beta_{2} T_{1}-r_{1}}{r_{1}}\right)^{2} .
\end{aligned}
$$


Substitute (33) and (34) into (23); then, it can be written as follows:

$$
r_{1}\left(\beta_{4} N_{1}-f\right)^{2}+f\left(\beta_{2} T_{1}-r_{1}\right)^{2} \approx 2 r_{1} f R .
$$

Due to equations,

$$
\begin{aligned}
& \left\langle\left(\beta_{4} N_{2}-f\right)^{2}\right\rangle_{t} \approx f R, \\
& \left\langle\left(r_{1}-\beta_{2} T_{1}\right)^{2}\right\rangle_{t} \approx r_{1} R,
\end{aligned}
$$

thus, we obtain that

$$
\begin{aligned}
m(R) & \approx \frac{1}{2} r_{1} D_{2}-\frac{1}{2} f D_{1}-b_{1} r_{1} f R, \\
\sigma^{2}(R) & \approx\left(f D_{1}-r_{1} D_{2}\right) R .
\end{aligned}
$$

When $R \longrightarrow 4.3782$, the boundary is nonsingular; when $R \longrightarrow \infty$, it is the second kind of singular boundary. The diffusion index $a=1$, the drift index $\beta=1, m(+\infty)<0$. Therefore, the nontrivial stationary probability density exists. And its steady-state probability density function $p(r)$ satisfies the following FPK equation:

$$
\frac{\mathrm{d}}{\mathrm{d} r}[m(r) p(r)]-\frac{1}{2} \frac{\mathrm{d}_{2}}{\mathrm{~d} r^{2}}\left[\sigma^{2}(r) p(r)\right]=0 .
$$

Whence,

$$
\begin{aligned}
m(r) & =\left.m(R)\right|_{R=r}, \\
\sigma^{2}(r) & =\left.\sigma^{2}(R)\right|_{R=r} .
\end{aligned}
$$

Solving the FPK equation, we then have

$$
p(r)=C \frac{T(r)}{g(r)} \exp \left[\int_{0}^{r} \frac{r_{1} f\left(r_{1} D_{2}-f D_{1}\right) T(x)-2 r_{1} b_{1} f g(x)}{\left(f D_{1}-r_{1} D_{2}\right) g(x)} \mathrm{d} x\right],
$$

where $C$ is a normalization constant, and

$$
\begin{aligned}
C & =\frac{C_{1} r_{1} f}{f D_{1}-r_{1} D_{2}}, \\
\frac{\mathrm{d} g(r)}{\mathrm{d} r} & =r_{1} \oint \frac{\beta_{4} N_{1}}{T_{1}\left(\beta_{4} N_{1}-f\right)} \mathrm{d} T_{1} .
\end{aligned}
$$

Hence, equation (40) can be simplified as follows:

$$
\begin{gathered}
p(r)=C T(r) \exp (v r), \\
v=\frac{-2 r_{1} b_{1} f}{f D_{1}-r_{1} D_{2}} .
\end{gathered}
$$

Suppose $R=r$, the conditional probability density of random process $N_{1}$ is $p\left(N_{1} / r\right)$; then, the joint probability density of $R$ and $N_{1}$ can be expressed as follows:

$$
p\left(r_{1}, N_{1}\right)=\frac{p(r)}{\left|N_{1}\left(r_{1}-\beta_{2} T_{1}\right)\right| T(r)} .
$$

As a result, we obtain the stationary probability density of $N_{1}$ and $T_{1}$ :

$$
p\left(N_{1}, T_{1}\right)=\frac{C}{N_{1} T_{1}} \exp (v r)
$$

Thus, we get the steady-state probability densities of $N_{1}$ and $T_{1}$, respectively. They are

$$
\begin{aligned}
& p\left(N_{1}\right)=\int_{0}^{\infty} p\left(N_{1}, T_{1}\right) \mathrm{d} T_{1}=\int_{0}^{\infty} \frac{C}{N_{1} T_{1}} \exp (v r) \mathrm{d} T_{1}, \\
& p\left(T_{1}\right)=\int_{0}^{\infty} p\left(N_{1}, T_{1}\right) \mathrm{d} N_{1}=\int_{0}^{\infty} \frac{C}{N_{1} T_{1}} \exp (v r) \mathrm{d} N_{1} .
\end{aligned}
$$

3.2.2. Mean First-Passage Time. When people are suffering from tumor, we hope that the number of tumor cells go to zero. However, according to the calculation in the above section, we know that the steady-state probability density of tumor cells is present, which indicates that tumor cells will not be extinct under natural state, but the effects of random factors may change this. Therefore, it is practical to study the average first-passage time $\beta$ from the coexistence equilibrium point to the tumor-free equilibrium point, and the Pontryagin equation is satisfied that

$$
1+m\left(x_{0}\right) \mid \frac{\mathrm{d} \beta}{\mathrm{d} x_{0}}+\frac{1}{2} \sigma^{2}\left(x_{0}\right) \frac{\mathrm{d}^{2} \beta}{\mathrm{d} x_{0}^{2}}=0
$$

where

$$
\begin{gathered}
m\left(x_{0}\right)=\left.m(R)\right|_{R=x_{0}}, \\
\sigma^{2}\left(x_{0}\right)=\left.\sigma^{2}(R)\right|_{R=x_{0}} .
\end{gathered}
$$

From the above studies, we know that $x_{l}$ is nonsingular and $x_{s}$ is natural. According to the constant variation method, $\beta$ can be written as follows:

$$
\beta\left(x_{0}\right)=2 \int_{x_{0}}^{x_{s}} \psi^{-1}(\mu)\left[\int_{x_{l}}^{\mu} \frac{1}{\sigma^{2(v)}} \psi(v)+C_{1}\right] \mathrm{d} \mu,
$$

where $C_{1}$ is the integral constant, and

$$
\psi(v)=\exp \left[\int \frac{2 m(v)}{\sigma^{2}(v)} \mathrm{d} v\right]
$$

\section{Numerical Simulation}

Refer to the experimental data of article [20-29] (Table 1) and the analysis of stability of equilibria in the previous section, and we obtain that system (19) of the tumor-free equilibrium is stable, and the corresponding critical state is denoted by $x_{s}$. Calculate based on Table 1 and reference to the Figure 1, we know the result of coexisting equilibrium belongs to the red area when the $m$ value is $-0.8,-0.9$, and -1.0. Namely, system (19) has only one stable coexistence equilibrium, and the corresponding critical state is denoted by $x_{l}$. As a result, $x_{l}$ is nonsingular, $x_{s}$ is natural, and the expression of the first-passage time from $x_{l}$ to $x_{s}$ satisfies equation (46). In order to investigate the effect of the Allee 
TABLE 1: The definitions and corresponding value of variables in models (2) and (19).

\begin{tabular}{|c|c|c|c|}
\hline Parameter & Definition & Value & Source \\
\hline$s$ & Constant source of effector cells & 0.33 & {$[21,23]$} \\
\hline$\rho$ & Maximum effector cells recruitment rate by tumor cells & 0.01 & {$[20,21]$} \\
\hline$\alpha$ & Steepness coefficient of the effector cells recruitment & 0.3 & {$[20,22]$} \\
\hline$\beta_{1}$ & Effector cells inactivation rate by tumor cells & 1.0 & {$[23,24]$} \\
\hline$d_{1}$ & Death rate of effector cells & 0.2 & {$[20,29]$} \\
\hline$r_{1}$ & Normal cells' growth rate & 1.0 & {$[20,25]$} \\
\hline$r_{2}$ & Tumor growth rate & 1.5 & {$[26,27]$} \\
\hline$b_{2}$ & Reciprocal of tumor carrying capacity & 1.0 & {$[20,28]$} \\
\hline$b_{1}$ & Reciprocal of normal cells' carrying capacity & 1.0 & {$[20,28]$} \\
\hline$\beta_{2}$ & Fractional normal cells killed by tumor cells & 1.0 & {$[23,24]$} \\
\hline$\beta_{3}$ & Fractional tumor cells killed by effector cells & 0.5 & {$[23,24]$} \\
\hline$\beta_{4}$ & Inactivation rate of tumor cells owing competition with normal cells & 1.0 & {$[20,23]$} \\
\hline$m$ & Threshold value of Allee effect & $m<1$ & \\
\hline
\end{tabular}

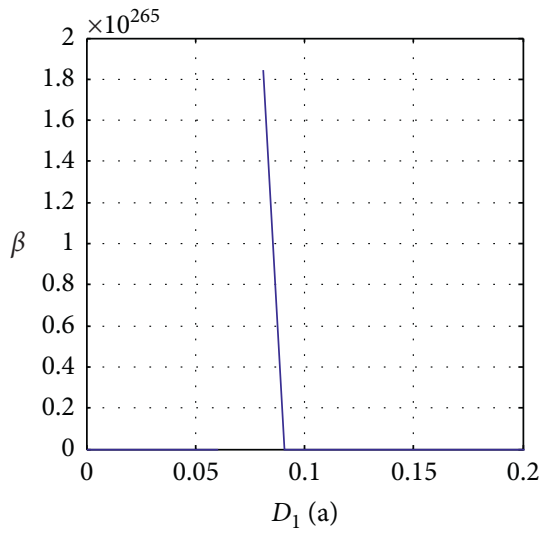

(a)

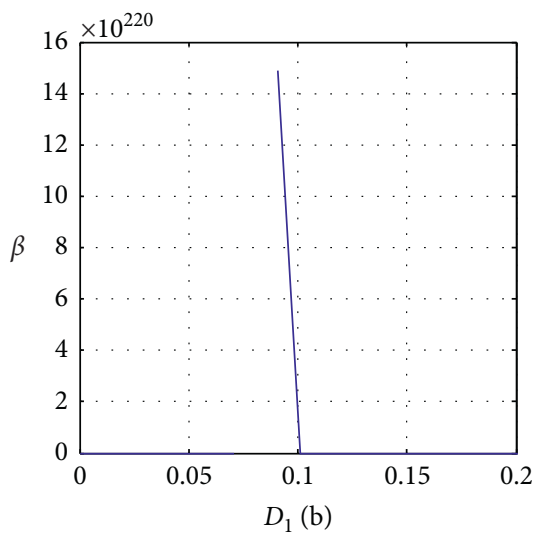

(b)

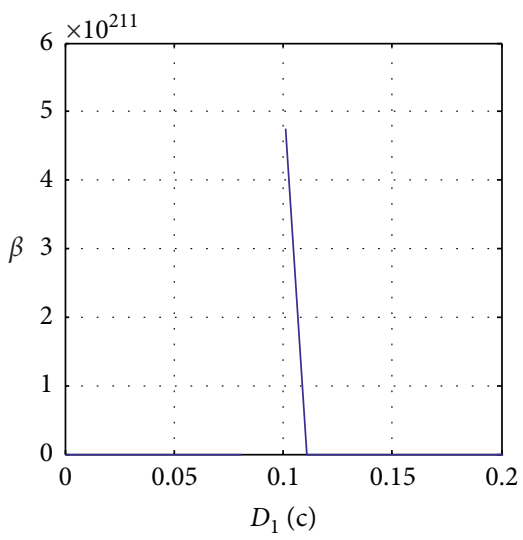

(c)

Figure 3: Mean first-passage time varies with $D_{1}$. (a) $m=-1.0$. (b) $m=-0.9$. (c) $m=-0.8$.

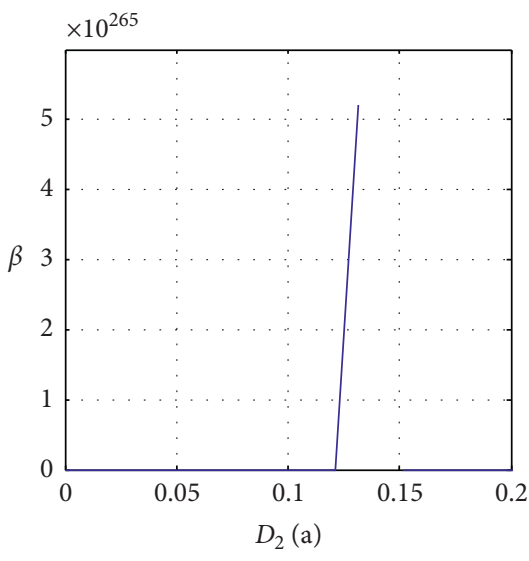

(a)

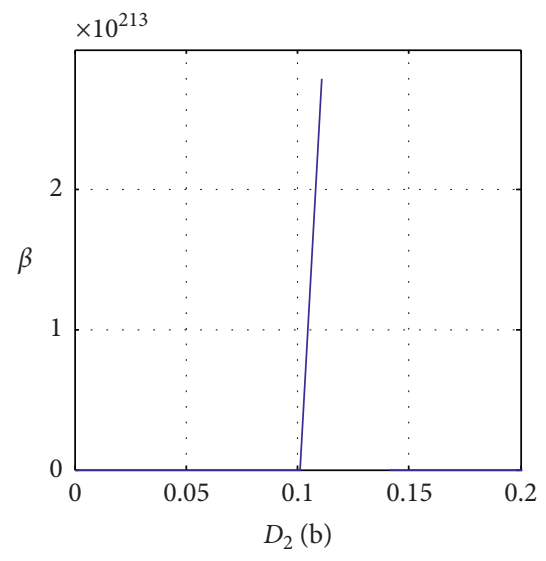

(b)

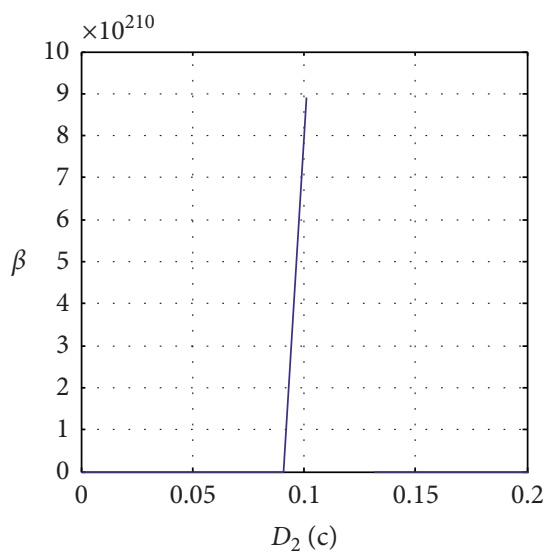

(c)

Figure 4: Mean first-passage time varies with $D_{2}$. (a) $m=-1.0$. (b) $m=-0.9$. (c) $m=-0.8$.

threshold and noise intensity on the first-passage time, we numerically simulate the above results and obtain Figures 3 and 4.
As can be seen from Figures 3 and 4, the time of first passage is limited only when the random intensity is within a certain range. This indicates that the existence of random 
factors does not cause the first-passage to occur, and only when the random intensity is within a certain range will the system cross from the coexistence equilibrium to the tumorfree equilibrium.

\section{Conclusions}

In this paper, we analyze the property of the deterministic tumor-immune model under the Allee effect, including the positivity and boundness. The results show that, in the condition of $d_{1}>\rho$, the solution of system (2) is positive and bounded. At the same time, we know that the Allee effect affects the growth and reproduction of cells in the tumorimmune system according to Figure 2. And the influence is most significant when the Allee threshold of tumor cells is in the range of -1.2 to -0.5 .

And in the meantime, we are devoted to explore the effect of random factors on the transition of the tumorimmune system from the stable coexistence equilibrium to the stable tumor-free equilibrium. Based on the method of stochastic averaging, we obtain the expressions of the steadystate probability density and the mean first-passage time. Through the numerical simulation about the expression of first-passage time, we know that the existence of random factors will not make the first-passage must happen, in other words, the random strength only within a certain range; the system will pass from the stable coexistence equilibrium point to the tumor-free equilibrium point. Our conclusions provide a possible method to change the number of tumor cells, which is expected to be helpful for the treatment of tumors.

\section{Data Availability}

The data used to support the findings of this study are included within the article.

\section{Conflicts of Interest}

The authors declare that there are no conflicts of interest regarding the publication of this paper.

\section{Acknowledgments}

This research was funded by the National Natural Science Foundation (11772002), Ningxia Higher Education Firstclass Discipline Construction Funding Project (NXYLXK2017B09), Major Special Project of North Minzu University (ZDZX201902), Open Project of the Key Laboratory of Intelligent Information and Big Data Processing of NingXia Province (2019KLBD008), "Light of the West" Talent Training Program of Chinese Academy of Sciences (XAB2016AW04), and Graduate Innovation Project of North Minzu University (YCX19130).

\section{References}

[1] B. J. Thompson, "YAP/TAZ: drivers of tumor growth, metastasis, and resistance to therapy," BioEssays, vol. 42, no. 5, Article ID 1900162, 2020.
[2] S. Du, N. Mccall, K. Park et al., "Blockade of tumor-expressed PD-1 promotes lung cancer growth," OncoImmunology, vol. 7, no. 4, Article ID e1408747, 2018.

[3] A. S. Pires, R. C. Marque, A. M. Abrantes et al., "Ascorbic acid chemosensitizes colorectal cancer cells and synergistically inhibits tumor growth," Frontiers in Physiology, vol. 9, p. 911, 2018.

[4] N. Chaoul, A. Tang, B. Desrues et al., "Lack of MHC class II molecules favors CD8+ T-cell infiltration into tumors associated with an increased control of tumor growth," Oncoimmunology, vol. 7, no. 3, Article ID e1404213, 2017.

[5] H. A. Villalobos, D. Bobbala, R. Kandhi et al., "SOCS1 inhibits migration and invasion of prostate cancer cells, attenuates tumor growth and modulates the tumor stroma," Prostate Cancer and Prostatic Diseases, vol. 20, no. 1, pp. 36-47, 2017.

[6] B. Volz, K. Kapp, D. Oswald et al., "A new family of potent TLR9 agonists, EnanDIM, which inhibit tumor growth in various syngeneic murine models," European Journal of Cancer, vol. 92, no. 1, pp. S5-S6, 2018.

[7] A. Adriana, B. Antonino, D. M. Noonan et al., "Contribution to tumor angiogenesis from innate immune cells within the tumor microenvironment: implications for immunotherapy," Frontiers in Immunology, vol. 9, p. 527, 2018.

[8] M. Preusser, A. S. Berghoff, C. Thallinger et al., "Cancer immune cycle: a video introduction to the interaction between cancer and the immune system," ESMO Open, vol. 1, no. 3, Article ID e000056, 2016.

[9] Y. Jin, Y. Hong, and C. Hong, "Molecular interactions of autophagy with the immune system and cancer," International Journal of Molecular Sciences, vol. 18, no. 8, pp. 1694-1703, 2017.

[10] R. Medzhitov and C. A. Janeway, "Innate immunity: the virtues of a nonclonal system of recognition," Cell, vol. 91, no. 3, pp. 295-298, 1997.

[11] J. Wang, D. Li, H. Cang et al., "Crosstalk between cancer and immune cells: role of tumor-associated macrophages in the tumor microenvironment," Cancer Medicine, vol. 8, no. 10, pp. 1-13, 2019.

[12] H. Simon, H. L. Christian, and K. Simone, "MiRNAs: dynamic regulators of immune cell functions in inflammation and cancer," Cancer Letters, vol. 431, pp. 11-21, 2018.

[13] E. Naghmehossadat, F. L. Tamara, and H. K. Phillip, "Dynamic adaptation of tumor immune response with nivolumab demonstrated by 18F-FDG PET/CT," Clinical Nuclear Medicine, vol. 43, no. 2, pp. 114-116, 2018.

[14] L. Pang, S. Liu, X. Zhang et al., "Mathematical modeling and dynamic analysis of anti-tumor immune response," Journal of Applied Mathematics and Computing, vol. 62, no. 1-2, pp. 473-488, 2020.

[15] S. Khajanchi, "Chaotic dynamics of a delayed tumor-immune interaction model," International Journal of Biomathematics, vol. 13, no. 2, pp. 1039-1091, 2020.

[16] S. Terry, P. Savagner, S. Ortiz-Cuaran et al., "New insights into the role of EMT in tumor immune escape," Molecular Oncology, vol. 11, no. 7, pp. 824-846, 2017.

[17] B. Katrin, H. Hatzikirou, V. B. Anja et al., "An emerging Allee effect is critical for tumor initiation and persistence," PLoS Computational Biology, vol. 11, no. 9, Article ID e1004366, 2015.

[18] A. Konstorum, T. Hillen, and J. Lowengrub, "Feedback regulation in a cancer stem cell model can cause an Allee effect," Bulletin of Mathematical Biology, vol. 78, no. 4, pp. 754-785, 2016. 
[19] P. A. Stephens, W. J. Sutherland, and R. P. Freckleton, "What is the Allee effect?" Oikos, vol. 87, no. 1, pp. 185-190, 1999.

[20] L. G. D. Pillis and A. Radunskaya, "The dynamics of an optimally controlled tumor model: a case study," Mathematical and Computer Modelling, vol. 37, no. 11, pp. 12211244, 2003.

[21] L. G. D. Pillis and A. Radunskaya, "A mathematical tumor model with immune resistance and drug therapy: an optimal control approach," Journal of Theoretical Medicine, vol. 3, no. 2, pp. 79-100, 2001

[22] V. A. Kuznetsov, I. A. Makalkin, M. A. Taylor, and A. S. Perelson, "Nonlinear dynamics of immunogenic tumors: parameter estimation and global bifurcation analysis," Bulletin of Mathematical Biology, vol. 56, no. 2, pp. 295-321, 1994.

[23] J. C. Panetta, "A mathematical model of periodically pulsed chemotherapy: tumor recurrence and metastasis in a competitive environment," Bulletin of Mathematical Biology, vol. 58, no. 3, pp. 425-447, 1996.

[24] S. Michelson and J. T. Leith, "Host response in tumor growth and progression," Invasion \& Metastasis, vol. 16, no. 4-5, pp. 235-246, 1996.

[25] S. Kusama, J. S. Spratt, W. L. Donegan, F. R. Watson, and C. Cunningham, "The gross rates of growth of human mammary carcinoma," Cancer, vol. 30, no. 2, pp. 594-599, 1972.

[26] V. C. Arnerl, S. O. Emdin, B. Lundgren et al., "Breast carcinoma growth rate described by mammographic doubling time and S-phase fraction. Correlations to clinical and histopathologic factors in a screened population," Cancer, vol. 70, no. 7, pp. 1928-1934, 1992.

[27] N. Gunduz, B. Fisher, and E. A. Saffer, "Effect of surgical removal on the growth and kinetics of residual tumor," Cancer Research, vol. 39, no. 10, pp. 3861-3865, 1979.

[28] J. D. Ford, "Organizational change as shifting conversations," Journal of Organizational Change Management, vol. 12, no. 6, pp. 480-500, 1999.

[29] L. G. D. Pillis, W. Gu, K. R. Fister et al., "Chemotherapy for tumors: an analysis of the dynamics and a study of quadratic and linear optimal controls," Mathematical Biosciences, vol. 209, no. 1, pp. 292-315, 2007. 